\title{
STAR CLUSTER SIMULATIONS: THE STATE OF THE ART
}

\author{
SVERRE J. AARSETH \\ Institute of Astronomy, Madingley Road, Cambridge, England
}

\begin{abstract}
This paper concentrates on four key tools for performing star cluster simulations developed during the last decade which are sufficient to handle all the relevant dynamical aspects. First we discuss briefly the Hermite integration scheme which is simple to use and highly efficient for advancing the single particles. The main numerical challenge is in dealing with weakly and strongly perturbed hard binaries. A new treatment of the classical Kustaanheimo-Stiefel two-body regularization has proved to be more accurate for studying binaries than previous algorithms based on divided differences or Hermite integration. This formulation employs a Taylor series expansion combined with the Stumpff functions, still with one force evaluation per step, which gives exact solutions for unperturbed motion and is at least comparable to the polynomial methods for large perturbations. Strong interactions between hard binaries and single stars or other binaries are studied by chain regularization which ensures a non-biased outcome for chaotic motions. A new semi-analytical stability criterion for hierarchical systems has been adopted and the long-term effects on the inner binary are now treated by averaging techniques for cases of interest. These modifications describe consistent changes of the orbital variables due to large Kozai cycles and tidal dissipation. The range of astrophysical processes which can now be considered by $\mathrm{N}$-body simulations include tidal capture, circularization, mass transfer by Roche-lobe overflow as well as physical collisions, where the masses and radii of individual stars are modelled by synthetic stellar evolution.
\end{abstract}

Key words: Numerical methods, KS-regularization, N-body problem

\section{Introduction}

The study of self-gravitational $N$-body systems by direct integration poses many technical challenges which must be addressed. However, progress during the last decade now enables such problems to be tackled with confidence. In this personal review of recent developments, we concentrate on four main numerical tools which appear to be sufficient for the task in hand. The corresponding algorithms may be summarized under the following headings:

- Hermite integration

- Two-body regularization

- Chain regularization

- Hierarchical systems

These topics are discussed briefly in the subsequent sections, together with an outline of current applications. Given adequate tools, a massive effort is still required in order to develop an efficient star cluster simulation code but these aspects are beyond the scope of the present contribution.

\section{Hermite Scheme}

Although the Hermite integration scheme was developed for the special-purpose HARP computer (Makino 1991), it is also proving highly effective for standard workstations as well as conventional supercomputers. Since coding is now somewhat simpler than for the traditional divided difference formulation (Ahmad and Cohen 1973, Aarseth 1985), it should be considered the method of choice for direct 
$\mathrm{N}$-body simulations. It may also be remarked that Hermite integration is actually more accurate than divided differences for the same order.

The main idea is again to employ a fourth-order force polynomial but now the two first terms are evaluated by explicit summation over all $N$ particles, thereby enabling two corrector terms to be formed. At first sight it may seem rather expensive to extend the full summation to the force derivative since this also requires prediction of velocities. However, simplicity as well as increased accuracy combine to outweigh the drawback of extra operations, particularly if block-step predictions are introduced. We expand a Taylor series for the force $\mathbf{F}$ and its first derivative $\mathbf{F}^{(1)}$ for each particle up to the third derivative about the reference time $t$ as

$$
\begin{aligned}
\mathbf{F} & =\mathbf{F}_{0}+\mathbf{F}_{0}^{(1)} t+\frac{1}{2} \mathbf{F}_{0}^{(2)} t^{2}+\frac{1}{6} \mathbf{F}_{0}^{(3)} t^{3}, \\
\mathbf{F}^{(1)} & =\mathbf{F}_{0}^{(1)}+\mathbf{F}_{0}^{(2)} t+\frac{1}{2} \mathbf{F}_{0}^{(3)} t^{2} .
\end{aligned}
$$

Substituting $\mathbf{F}_{0}^{(2)}$ from (2) into (1) and simplifying then yields the third derivative corrector

$$
\mathbf{F}_{0}^{(3)}=\left(2\left(\mathbf{F}_{0}-\mathbf{F}\right)+\left(\mathbf{F}_{0}^{(1)}+\mathbf{F}^{(1)}\right) t\right) \frac{6}{t^{3}} .
$$

Similarly, substituting (3) into (1) gives the second derivative corrector

$$
\mathbf{F}_{0}^{(2)}=\left(-3\left(\mathbf{F}_{0}-\mathbf{F}\right)-\left(2 \mathbf{F}_{0}^{(1)}+\mathbf{F}^{(1)}\right) t\right) \frac{2}{t^{2}}
$$

Using $F_{0}$ and $F_{0}^{(1)}$ evaluated at the beginning of a time-step, the coordinates and velocities are first predicted to order $\mathbf{F}^{(1)}$ for all particles. Following determination of the new $\mathbf{F}$ and $\mathbf{F}^{(1)}$ by summation over all the contributions, the two higher derivatives are obtained by (3) and (4). This gives rise to corrector terms for coordinates and velocities given by

$$
\begin{aligned}
\Delta \mathbf{r}_{i} & =\frac{1}{24} \mathbf{F}_{0}^{(2)} \Delta t^{4}+\frac{1}{120} \mathbf{F}_{0}^{(3)} \Delta t^{5}, \\
\Delta \mathbf{v}_{i} & =\frac{1}{6} \mathbf{F}_{0}^{(2)} \Delta t^{3}+\frac{1}{24} \mathbf{F}_{0}^{(3)} \Delta t^{4}
\end{aligned}
$$

Given the high-order derivatives, individual time-steps can now be assigned in the usual way from some suitable convergence criterion.

The overheads of predicting $N$ coordinates and velocities at each time-step can be reduced considerably by adopting so-called hierarchical time-steps (McMillan 1986), where the indicated values are truncated to be factor 2 commensurate. The apparent inefficiency of just a few particles sharing the same (small) step and yet requiring one full prediction is compensated by having a distribution of discrete levels (typically 16 for $N \simeq 10^{4}$ ) such that the number of predictions is significantly reduced with respect to the continuous case (say by factor of 100). This scheme is particularly suitable for the special-purpose HARP computers but lends itself equally well to other architectures, including parallel supercomputers (Spurzem 
1998). Somewhat surprisingly, the workstation code NBODY6 which is based on the Ahmad-Cohen (1973) neighbour scheme (Makino and Aarseth 1992, Aarseth 1994) is in fact slightly faster and more stable than the older NBODY5 code for $N=1000$ single particles and the same number of steps.

\section{Two-Body Regularization}

The early 1970's saw the introduction of the Kustaanheimo-Stiefel (1965) regularization for treating close encounters and hard binaries in $N$-body simulations (Bettis and Szebehely 1972, Aarseth 1972) and the elegant KS method has proved to be very resilient. However, even a regularized two-body solution is subject to small but systematic errors when studied over long times. In order to avoid this undesirable feature, the concept of energy stabilization has been tried for weak perturbations (Aarseth 1985). Although this procedure ensures that the orbit is constrained to have the correct energy arising from the perturbation, the corresponding angular momentum is no longer conserved so well.

The subsequent exploitation of adiabatic invariance (Mikkola and Aarseth 1996) by the so-called slow-down principle tends to alleviate this imperfection since now one KS orbit may represent a number of physical periods by augmenting the perturbation itself and neglecting short-period effects. As for the earlier claim that a time-symmetric KS method would be superior (Funato et al. 1996), it now appears that the requirement of variable time-steps for perturbed orbits cannot be accommodated (Kokubo et al. 1998). So far there is no evidence that the resulting eccentricities of cluster binaries studied by the stabilization scheme cannot be trusted, especially bearing in mind that the long-term evolution of most binaries is predominantly subject to discrete changes of a random nature. The case of long-lived hierarchical systems deserves special consideration, however, but here additional effects should also be considered, as discussed in a subsequent section.

An alternative KS regularization scheme has been presented recently (Mikkola and Aarseth 1998) which achieves a high accuracy without extra cost. This new approach is based on the idea of a truncated Taylor series, where additional correction terms represent the neglected higher orders and which yields exact solutions in the unperturbed case. The new algorithm is again of Hermite type and will be outlined in the following.

First, coordinates and velocities of the perturbers are predicted in the usual way (i.e. to first order), whereas the regularized coordinates and velocities $\left(U, U^{\prime}\right)$ are predicted to highest order. Here $U^{(4)}, U^{(5)}$ include the modified Stumpff (1962) functions

$$
\tilde{c}_{n}(z)=n ! \sum_{k=0}^{\infty} \frac{(-z)^{k}}{(n+2 k) !},
$$

where the argument is related to the time-step by $z=-\frac{1}{2} h \Delta \tau^{2}$ and $h$ is the specific binding energy. These coefficients only deviate slightly from unity and a twelfthorder expansion (re-evaluated every step) appears sufficient. After transforming 
the physical coordinates and velocities to global values, the predictor cycle is completed by evaluating the perturbing acceleration $\mathbf{F}$ as well as its explicit derivative $\dot{\mathbf{F}}$.

Because of the insufficient accuracy of the predicted deviation from unperturbed motion at the end of a step, the corrector cycle employs an iteration. Setting $\Omega=-\frac{1}{2} h$, the basic equation of motion takes the familiar form

$$
\mathbf{U}^{(2)}=-\mathbf{\Omega U}+\frac{1}{2} r \mathcal{L}^{T} \mathbf{F},
$$

where $\mathcal{L}(\mathbf{U})$ is a $4 \times 3$ linear matrix and $r=\mathbf{U} \cdot \mathbf{U}$ is the separation. We express the new KS acceleration and its derivative (where $\mathbf{F}^{\prime}=r \mathbf{F}$ ) at the start of a step as

$$
\begin{aligned}
& \mathbf{U}_{0}^{(2)}=-\Omega_{0} \mathbf{U}_{0}+\mathbf{f}_{\mathbf{0}}^{(2)}, \\
& \mathbf{U}_{0}^{(3)}=-\Omega_{0} \mathbf{U}_{0}^{\prime}+\mathbf{f}_{\mathbf{0}}^{(3)},
\end{aligned}
$$

where $\mathrm{f}_{0}^{(2)}=\frac{1}{2} r \mathbf{Q}$, with $\mathbf{Q}=\mathcal{L}^{\mathbf{T}} \mathbf{F}$, is the perturbed force contribution evaluated after the previous predictor cycle.

The two next Taylor series terms are constructed from the Hermite scheme. Using the current value of $h$ (and $\Omega$ ), predicted to fourth order, we form the new perturbative functions at the end of the step

$$
\begin{aligned}
& \mathbf{f}^{(\mathbf{2})}=\left(\boldsymbol{\Omega}_{0}-\boldsymbol{\Omega}\right) \mathbf{U}+\frac{1}{2} r \mathbf{Q}, \\
& \mathbf{f}^{(\mathbf{3})}=\left(\boldsymbol{\Omega}_{0}-\boldsymbol{\Omega}\right) \mathbf{U}^{\prime}-\boldsymbol{\Omega}^{\prime} \mathbf{U}+\frac{1}{2} r^{\prime} \mathbf{Q}+\frac{1}{2} r \mathbf{Q}^{\prime},
\end{aligned}
$$

from which the corrector derivatives $f_{0}^{(4)}, f_{0}^{(5)}$ are recovered by the Hermite rule (Makino 1991).

The expressions for $\mathrm{U}_{0}^{(4)}$ and $\mathrm{U}_{0}^{(5)}$ are readily formed in analogy with Eqs. (8) and (9) which yield

$$
\begin{aligned}
& \mathbf{U}_{0}^{(4)}=-\Omega_{0} \mathbf{U}_{0}^{(2)}+\mathbf{f}_{0}^{(4)}, \\
& \mathbf{U}_{0}^{(5)}=-\Omega_{0} U_{0}^{(3)}+\mathbf{f}_{0}^{(5)} .
\end{aligned}
$$

From Eqs. (8) - (13), the provisional solution for $U, \mathbf{U}^{\prime}$ is then obtained by the general expression (cf. Mikkola and Aarseth 1998), which contains the Stumpff functions. The treatment of the energy remains the same as for standard Hermite based on $\Omega^{\prime}=-\mathbf{U}^{\prime} \cdot \mathbf{Q}$ and the physical time is obtained from integrating $t^{\prime}=\mathrm{U} \cdot \mathrm{U}$ which also involves Stumpff functions. Substituting for $\mathbf{U}^{(2)}$, we write the second derivative as

$$
\mathbf{\Omega}^{(2)}=\Omega_{0} \mathbf{U} \cdot \mathbf{Q}-\mathbf{f}^{(2)} \cdot \mathbf{Q}-\mathbf{U}^{\prime} \cdot \mathbf{Q}^{\prime} .
$$

The two corrector terms constructed from $\Omega^{\prime}$ and $\Omega^{(2)}$ are added to the predicted value without any Stumpff functions to yield an improved solution for $\Omega$ at the start of the next iteration or at the end point. 
Subsequent iterations repeat the procedure above, starting from Eq. (10) without re-evaluating the physical perturbation and its derivative. Thus the new values of Eqs. (10) and (11) are based on the improved solution for $\mathbf{U}, \mathbf{U}^{\prime}$ and $r, r^{\prime}$, as well as the new $\Omega$. In the present treatment, one iteration yields a significant improvement for modest perturbations and experience so far indicates that this may also be sufficient for strong interactions because of the shortening of the stepsize $\Delta \tau$ (cf. Aarseth 1994).

The corrector cycle ends by specifying new derivatives for use in the next prediction, as well as saving the perturbative derivatives (10) and (11) required for the Hermite scheme. This is completed by re-initializing Eqs. (8) and (9) at the end point, substituting $f^{(2)}, f^{(3)}$ as well as the iterated values of $\Omega^{\prime}$ and $\Omega^{(2)}$. It is advantageous to employ the corrected values of $r$ and $r^{\prime}$ for this purpose; the re-evaluation of $\mathbf{f}^{(2)}$ and $\mathbf{f}^{(3)}$ is fast and also benefits the final quantities $\mathbf{U}^{(2)}$ and $\mathrm{U}^{(3)}$ to be used in the next prediction. A more accurate expression of the fourth $\mathrm{KS}$ derivative at the end of the interval is obtained by including the next order by

$$
\mathbf{U}^{(4)}=\mathbf{U}_{0}^{(4)}+\mathbf{U}_{0}^{(5)} \Delta \tau,
$$

and similarly for the third derivative of the energy,

$$
\Omega^{(3)}=\Omega_{0}^{(3)}+\Omega^{(4)} \Delta \tau \text {. }
$$

The above scheme has been implemented in the state of the art codes NBODY4 and NBODY 6 and has proved itself in large-scale simulations. Accuracy tests obtained by a toy code shows that high accuracy can be obtained with 30 steps per orbit for relatively weak perturbations, which is about half that required by the old stabilization scheme. A significant part of this gain is due to the modifications by the Stumpff coefficients, although the basic Taylor series (or Encke-type) formulation is also considerably more accurate than the standard method. The number of operations for a typical step is not much larger in the new method, including the overhead for the Stumpff functions and one iteration in the corrector. Hence the computational effort is less for typical calculations, although this depends on the actual number of perturbers. Finally, we remark that the Stumpff method also includes the slow-down scheme in exactly the same way as before.

\section{Chain Regularization}

The concept of chain regularization is simple, yet the mathematical formulation is quite technical and this has acted as an impediment to wider usage. However, it enables new types of problems to be studied and is therefore worth the extra effort. The basic idea is a generalization of three-body regularization (Aarseth and Zare 1974) which treats two perturbed KS solutions with respect to a common reference body, where each two-body solution is described by regular equations. Thus an extension to four participating bodies merely introduces one more perturbed KS solution, although the formalism is somewhat different (Mikkola and Aarseth 
1990). Once the step from three to four particles has been mastered, the general case becomes feasible (Mikkola and Aarseth 1993).

The essential feature of chain regularization is that dominant interactions along the chain itself are treated as perturbed KS solutions and all the other attractions are included as perturbations. Hence it becomes imperative to select the chain vectors in such a manner as to minimize the perturbations. Since we are dealing with dynamical interactions, the chain vectors need to be redrawn in response to changing configurations. Fortuitously, all the relevant decision-making constitute a minor overhead here since the integration is carried out by the high-order BulirschStoer (1966) scheme and a certain elasticity is tolerated as regards switching to more favourable chain vectors.

The equations of motion are derived from a regularized Hamiltonian of the form

$$
\Gamma^{\star}=g(H-E)
$$

where $H$ is expressed in terms of the coordinates and momenta and $E$ is the internal system energy. Here the function $g$ is given by the corresponding time transformation

$$
d t=g d \tau
$$

and choosing the inverse Lagrangian energy $(L=T+\Phi)$ ensures regular solutions for any chain separation $R_{k}$.

The treatment begins by selecting a compact subsystem of three or four particles; i.e. so-called $B+S$ or $B+B$ type. External perturbers are chosen in analogy with the KS implementation and the internal integration includes any perturbation effect which also tends to change the total energy according to its separate equation of motion. At the same time, the c.m. motions are advanced by the standard Hermite scheme with due attention to the slightly modified form of the corresponding acceleration which requires a differential correction.

The analogy with KS does not hold in one important respect since the chain membership may change before termination occurs. Thus an initial subsystem of four members may lose one member due to ejection, or an approaching perturber a single particle or binary - may be added. Alternatively, the membership may also change through physical collision. All the relevant corrections and re-initializations are performed in situ. Hence the use of chain variables is also highly beneficial for the evaluation of nearly singular quantities. Chain termination usually occurs when a binary becomes well separated from one or two other members in which case the binary is accepted for KS treatment, whereas the remaining membership is initialized by the Hermite scheme or even as a second KS system. The actual decision-making also takes into account the cluster environment and is therefore quite involved.

Cluster simulations of primordial binaries frequently involve interactions of two binaries where the size of one is much less than the other. In such cases even 
the powerful chain method becomes prohibitive because the shortest period is a small fraction of the local crossing time. Fortunately the principle of slow-down applied to weakly perturbed KS binaries can also be employed here (Mikkola and Aarseth 1996). This permits a consistent study of binaries with arbitrarily short periods which would otherwise have to be treated as inert systems. The implementation itself differs from the KS case since here we adjust the slow-down factor continuously according to the maximum apocentre perturbation exerted by the other chain members, rather than choosing an appropriate discrete level (factor of 2) at each apocentre passage.

Since the strong interactions studied by the chain method are usually of short duration, the simulation code only allows one such case to be considered at a time for technical reasons. However, there is provision for studying one triple as well as one quadruple system by unperturbed three-body (Aarseth and Zare 1974) and chain (Mikkola and Aarseth 1990) regularization. Given a few hundred critical events in a typical cluster simulation, the latter procedures are usually not needed but this may change with the addition of more primordial binaries.

\section{Hierarchical Systems}

The Solar neighbourhood contains many examples of multiple systems where the inner component of a binary is itself a binary, and levels of higher multiplicity also exist. Likewise, hard binaries in star clusters may acquire an outer component with sufficiently small eccentricity to be stable over many orbits. Hierarchical triples may be formed by the classical three-body capture mechanism in which the binary itself acts mainly as a point-mass. However, in clusters with significant binary populations such systems are more likely to form in strong interactions between two binaries since this involves two-body encounters. The second formation process was already identified in scattering experiments with colliding binaries which yielded a high percentage of positive outcomes (Mikkola 1983). Thus one way for such triples to become stable requires the impact parameter to exceed some critical value and yet be sufficiently small for the weakest binary to be disrupted, but other processes are also favoured, including exchange.

Given a newly formed hierarchical triple, the question of long-term stability naturally arises. Depending on the period ratio, the direct calculation of a perturbed inner binary can be quite time-consuming even with $\mathrm{KS}$ regularization. However, since the corresponding semi-major axis may not be subject to any secular effects it becomes possible to adopt the centre-of-mass approximation and thereby only neglect cyclical changes of the eccentricity. Various empirical criteria have been obtained by fitting the results of systematic three-body calculations for a restricted set of parameters (Harrington 1977, Eggleton and Kiseleva 1995). Based on these results, the so-called merger procedure has been employed for some time (Aarseth 1985). Thus provided the stability condition is satisfied, the inner binary is replaced by its combined mass to facilitate KS treatment of the outer orbit.

A more rigorous approach based on correspondence with the chaos boundary 
in the binary-tides problem (Mardling 1995) has yielded a semi-analytical stability criterion which holds for quite large mass ratios and arbitrary outer eccentricities (Mardling and Aarseth 1998). Here the critical outer pericentre distance is given in terms of the inner semi-major axis, $a_{i n}$, by

$$
R_{p}^{\text {out }}=C\left[\left(1+q_{\text {out }}\right) \frac{\left(1+e_{\text {out }}\right)}{\left(1-e_{\text {out }}\right)^{1 / 2}}\right]^{2 / 5} a_{\text {in }},
$$

where $q_{\text {out }}=m_{3} /\left(m_{1}+m_{2}\right)$ is the outer mass ratio, $e_{\text {out }}$ is the corresponding eccentricity and $C \simeq 2.8$ is determined empirically. This criterion is only valid for coplanar prograde motion and still ignores a weak dependence on the inner eccentricity. However, the general case of inclined orbits exhibit increased stability so that Eq. (19) represents an upper limit. Further tests suggests an inclination correction factor $f=1-0.3 i / 180$ (with $i$ in degrees) which has been adopted in practical simulations; this is also in qualitative agreement with the original stability condition for retrograde orbits (Harrington 1972). The merger treatment is only allowed while the pericentre condition is satisfied, after which the inner binary is re-initialized.

A further refinement is included when the outer component itself is a binary. In the case of a $B+B$ configuration, the smallest binary plays the role of the outer body in a triple. Since the corresponding chaos boundary is not very sensitive to a second extended object (Mardling 1991), we adopt an additional correction factor $f_{1}=f+0.1 \min \left(a_{i n} / a_{2}, a_{2} / a_{i n}\right)$, with $a_{2}$ representing the second semi-major axis. We also mention here that even double hierarchies may be formed, where a system of type $B+S$ or $B+B$ itself acquires an outer bound component. Such configurations do occur occasionally and procedures have therefore been developed for their special treatment.

The criterion (19) above is concerned with long-term stability and hence the absence of escape. However, it is also of interest to consider the possibility of exchange between the outer component and one member of the inner binary. According to classical developments (Zare 1977, Szebehely and Zare 1977), the critical value for exchange in a coplanar prograde triple is given by

$$
\left(c^{2} E\right)_{c r i t}=-\frac{G^{2} f^{2}(\rho) g(\rho)}{2\left(m_{1}+m_{2}+m_{3}\right)},
$$

where $\mathbf{c}$ is the angular momentum and the functions $f(\rho), g(\rho)$ are expressed in terms of the masses, with $\rho$ determined by iteration from a fifth-order algebraic equation for the collinear equilibrium points. Numerical tests show that the chaos boundary given by Eq. (19) lies above the exchange boundary when the masses are comparable and the latter only begins to overlap above $q_{o u t} \simeq 5$. Application of the exchange criterion is therefore less useful in practical calculations. We also note that once an exchange occurs the final evolution will inevitably lead to escape.

The long-term evolution of a hierarchical triple is characterized by cyclic oscillations of the inner eccentricity where the amplitude depends on the inclination. 
The so-called Kozai effect (Kozai 1962) has received much attention recently in connection with external planetary systems but there is also an early example from N-body simulations (van Albada 1968) which points to the relevance for star clusters. Various analytical tools have been employed in order to model this process in some detail, including tidal dissipation for high eccentricities (Mardling and Aarseth 1999). Among the useful quantities which can be calculated theoretically (Heggie 1995) are the time-scale for a complete oscillation, $T_{K}$, as well as its maximum value, $e_{\max }$.

Since the time-scale for the Kozai cycle is usually much greater than the Kepler period, the merger scheme for hierarchical triples lends itself particularly well to a semi-analytical treatment. At present only systems with $e_{\max }>0.8$ are considered since smaller amplitudes are less likely to result in tidal activity. We have used a double averaging procedure (Eggleton 1997, Mardling and Eggleton 1998) to calculate the evolution of such systems in terms of the inner Runge-Lenz vector and angular momentum vector. Thus some examples show that inclinations near $90^{\circ}$ may induce tidal circularization even if oblateness effects are included. Clearly further developments of this experimental approach is needed in order to improve the modelling of these complicated processes.

\section{Astrophysical Applications}

The realistic simulation of star cluster dynamics requires a variety of astrophysical processes to be considered. In particular, the implementation of consistent stellar evolution enables the study of mass loss and finite-size effects. This is an ongoing project which has been outlined elsewhere (Aarseth 1996) and now employs an improved description of Roche mass transfer and physical collisions (Tout et al. 1997). Particular emphasis has been devoted to the modelling of chaotic motions and tidal circularization which form a link between an initial binary distribution and the Roche stage (Mardling and Aarseth 1999). In particular, it is found that very high eccentricities $(e>0.999)$ are produced in stable hierarchies or by exchange and these in turn lead to orbital shrinkage by tidal dissipation. Primordial binaries also leave an imprint in the form of high-velocity escapers. At the same time, more general cluster simulations have yielded much insight into dynamical evolution (McMillan et al. 1992, Aarseth and Heggie 1998, Portegies Zwart et al. 1998).

The modelling of synthetic stellar evolution is based on fast look-up tables for the radius, luminosity and type as a function of the initial mass and age (cf. Tout et al. 1997). Instantaneous mass loss due to stellar winds or supernovae explosions are adopted for the advanced stages. An energy-conserving integration scheme is preserved by including relevant corrections and re-initializations. The standard open cluster model includes $10^{4}$ single stars with $5 \%$ primordial hard binaries. Once the most massive single stars have evolved, the binaries dominate the mass segregation and increase their central abundance significantly with increased disruption probability. Even so, the original binary population is not depleted preferentially such that there is always an energy source which prevents core 


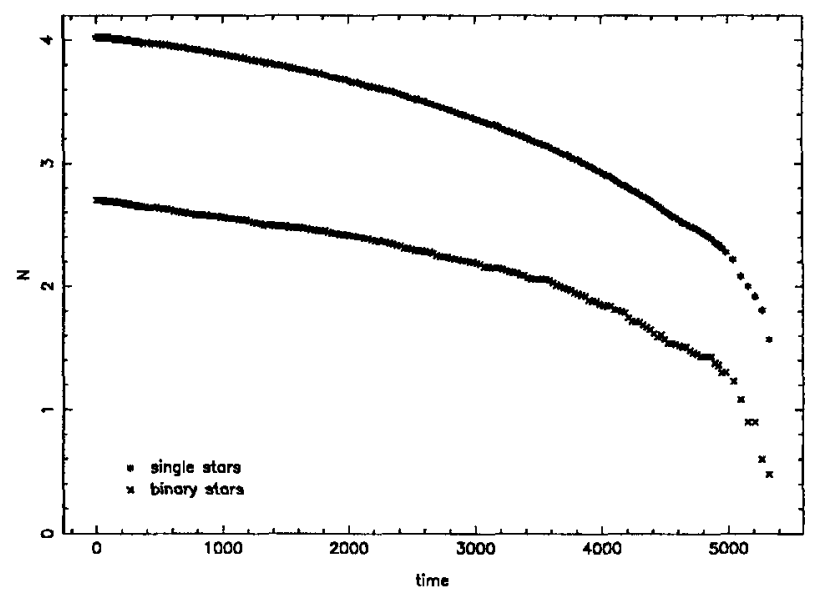

Fig. 1. Logarithmic plot of single stars and binaries as functions of time in $M y r$.

collapse. This behaviour is illustrated well in the figure which displays the bound membership.

In conclusion, the algorithms presented above have proved highly efficient for star cluster simulations. Hopefully these numerical tools will also play a part in future efforts involving more powerful hardware.

\section{References}

Aarseth, S.J.: 1972, Gravitational N-Body Problem, ed. Lecar, M. (Reidel), 373.

Aarseth, S.J.: 1985, Multiple Time Scales, ed. Brackbill, J.U. and Cohen, B.I. (Academic Press, New York), 377

Aarseth, S.J.: 1994, Galactic Dynamics and N-Body Simulations, ed. Contopoulos, G., Spyrou, N.K. and Vlahos, L. (Springer-Verlag), 277.

Aarseth, S.J.: 1996, Dynamical Evolution of Star Clusters, ed. Hut, P. and Makino, J. (Kluwer), 161. Aarseth, S.J. and Heggie, D.C.: 1998, Mon. Not. R. astr. Soc., $297,794$.

Aarseth, S.J. and Zare, K.: 1974, Celest. Mech., 10, 185.

Ahmad, A. and Cohen, L.: 1973, J. Comput. Phys., 12, 389.

van Albada, T.S.: 1968, Bull. Ast. Inst. Neth., 19, 479.

Bettis, D.G. and Szebehely, V.G.: 1972, Gravitational N-Body Problem, ed. Lecar, M. (Reidel), 388.

Bulirsch, R. and Stoer, J.: 1966, Num. Math., 8, 1.

Eggleton, P.P.: 1997, personal communication.

Eggleton, P.P. and Kiseleva, L.K.: 1995, Astrophys. J., 455, 640.

Funato, Y., Hut, P., McMillan, S. and Makino, J.: 1996, A.J., 112, 1697.

Harrington, R.S.: 1972, Celest. Mech., 6, 322.

Harrington, R.S.: 1977, A.J., 82, 753.

Heggie, D.C.: 1995, personal communication.

Kokubo, E., Yoshinaga, K. and Makino, J.: 1998, Mon. Not. R. astr. Soc., 297, 1067.

Kozai, Y. 1962, A.J., 67, 591.

Kustaanheimo, P. and Stiefel, E.: 1965, J. Reine Angew. Math., 218, 204.

Makino, J.: 1991, Astrophys. J., 369, 200. 
Makino, J. and Aarseth, S.J.: 1992, Publ. Astron. Soc. Japan, 44, 141.

Mardling, R.A.: 1991, Ph.D. Thesis, Monash University.

Mardling, R.A.: 1995, Astrophys. J., 450, 722.

Mardling, R. and Aarseth, S.: 1998, Proceedings NATO ASI, Maratea, ed. Roy, A.E., Steves, B. and Barnett, A.D. (Kluwer), in press.

Mardling, R.A. and Aarseth, S.J.: 1999, submitted.

Mardling, R.A. and Eggleton, P.P.: 1998, in preparation.

McMillan, S.L.W.: 1986, The Use of Supercomputers in Stellar Dynamics ed. Hut, P. and McMillan, S., (Springer-Verlag), 156.

McMillan, S.L.W., Hut, P. and Makino, J.: 1990, Astrophys. J., 362, 522.

Mikkola, S. 1983, Mon. Not. R. astr. Soc., 203, 1107.

Mikkola, S. and Aarseth, S.J.: 1990, Celest. Mech. Dyn. Astron., 47, 375.

Mikkola, S. and Aarseth, S.J.: 1993, Celest. Mech. Dyn. Astron., 57, 439.

Mikkola, S. and Aarseth, S.J.: 1996, Celest. Mech. Dyn. Astron., 64, 197.

Mikkola, S. and Aarseth, S.J.: 1998, New Astron., 3, 309.

Portegies Zwart, S., Hut, P., Makino, J. and McMillan, S.L.W.: 1998, Astron. Astrophys., 337, 363.

Spurzem, R.: 1999, The Journal of Computational and Applied Mathematics, ed. Riffert, H. and Werner, K. (Elsevier), in press.

Szebehely, V. and Zare, K.: 1977, Astron. Astrophys., 58, 145.

Stumpff, K.: 1962, Himmelsmechanik, Band I, (VEB, Berlin).

Tout, C.A., Aarseth, S.J., Pols, O.R. and Eggleton, P.P.: 1997, Mon. Not. R. astr. Soc., 291, 732.

Zare, K.: 1977, Celest. Mech., 16, 35. 